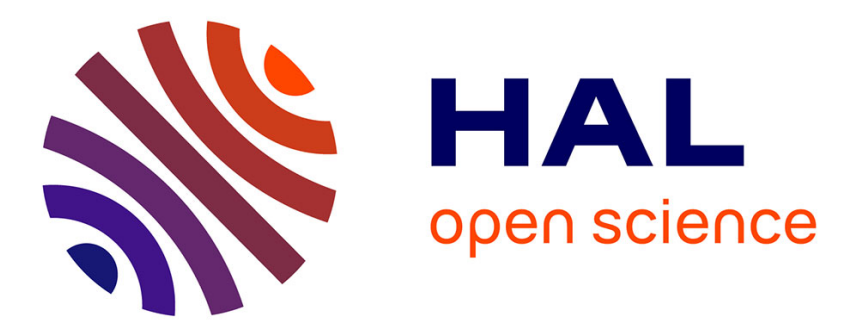

\title{
Un montant de naos au nom d'Amasis consacré au dieu Ptah
}

Christophe Thiers

\section{To cite this version:}

Christophe Thiers. Un montant de naos au nom d'Amasis consacré au dieu Ptah. Chr. Zivie-Coche, I. Guermeur. " Parcourir l'éternité ". Hommages à Jean Yoyotte, Brepols, pp.981-989, 2012, BEHE 156/2. halshs-00957245

\section{HAL Id: halshs-00957245 https://shs.hal.science/halshs-00957245}

Submitted on 13 Feb 2019

HAL is a multi-disciplinary open access archive for the deposit and dissemination of scientific research documents, whether they are published or not. The documents may come from teaching and research institutions in France or abroad, or from public or private research centers.
L'archive ouverte pluridisciplinaire HAL, est destinée au dépôt et à la diffusion de documents scientifiques de niveau recherche, publiés ou non, émanant des établissements d'enseignement et de recherche français ou étrangers, des laboratoires publics ou privés. 


\title{
UN MONTANT DE NAOS AU NOM D'AMASIS CONSACRE AU DIEU PTAH
}

\author{
Christophe THIERs ${ }^{*}$ \\ Heureux celui qui a pu pénétrer les causes secrètes des choses \\ Et qui a mis sous ses pieds toutes les craintes. \\ Virgile, Les Géorgiques II, 490.
}

Le montant de naos monolithel qui fait l'objet de la présente note a été saisi par la police chez un antiquaire de Louqsor en mars 1987 et a rejoint le dépôt de Gadaya (inv. 84) à l'intérieur de l'enceinte de Karnak. Le déménagement de ce magasin vers celui d'Abou Goud en mars-avril 2011 a permis de (re)découvrir plusieurs objets dignes du plus grand intérêt. Ce fragment de granodiorite assouanais ${ }^{2}$ est désormais conservé dans le magasin du Cheikh Labib; il mesure 173 (h) $\times 17,5$ (l) $\times 13,5$ (p) cm.

La partie arrière de ce montant gauche est cassée mais les côtés droit (extérieur) et gauche (intérieur) conservent leur face polie. Un léger retrait à l'extrémité du côté intérieur souligne la profondeur du montant à la limite de l'arrachement de la pierre. La partie supérieure conserve, sur $3 \mathrm{~cm}$, le départ du « linteau » du naos.

Une inscription soigneusement gravée (large de $16 \mathrm{~cm}$ ) court sur la presque totalité du montant, surmontée d'un signe du ciel et d'une ligne horizontale qui délimitait la décoration inférieure du linteau. Avant d'en examiner le contenu, on retiendra le rendu précis de certains signes et la volonté archaïsante bien connue pour cette période, par exemple :

- Jonc- $n$ h : les extrémités sont arrondies et la feuille de droite passe devant celle de gauche ${ }^{3}$.

\footnotetext{
* Directeur de recherche au CNRS, co-directeur du CFEETK-USR 3172.

1. Il m'est agréable de remercier Mansour Boraik, Directeur général des antiquités de Louqsor et de la Haute Égypte (Ministère d'État des Antiquités), co-directeur du CFEETK, pour l'autorisation de publication de ce monument, ainsi que Karima Dowi (CSA-CFEETK) pour les photographies et Solène Klein (stagiaire CNRS-CFEETK) pour le relevé épigraphique.

2. R. Klemm - D. D. Klemm, Stones \& Quarries in Ancient Egypt, Londres, 2008, p. 260-261

3. Cf. p. ex. Ph. Collombert, Le tombeau de Mérérouka. Paléographie (PalHiér 4), Le Caire 2004, p. 91-92, 227, § 159; H. G. Fischer, Egyptian Studies I, Varia, New York, 1976, pl. VII, fig. 4.
} 
- Pintade-nh $\aleph^{3}$ : malgré l'absence de caroncule, le contour général de l'oiseau le rend parfaitement identifiable, la protubérance sur la tête étant légèrement soulignée ${ }^{4}$.

- Support-? marquée ${ }^{5}$.

\section{Le texte}

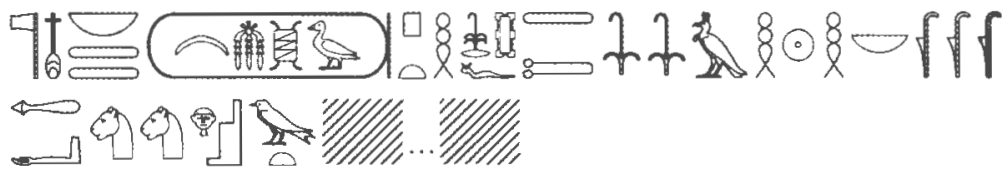

Le dieu parfait, maître du Double-Pays (Amasis fils de NeithX, [aimé] de Ptah qui est au Sud de son mur ${ }^{(\mathrm{s})}$, Tenen, l'Éternel, le maître des années ${ }^{(\mathrm{b})}$, dont la puissance est grande, qui est sur le Grand trône ${ }^{(c)}[\ldots]$.

(a) L'épithète traditionnelle de Ptah memphite «celui qui est au Sud de son mur » (M. Sandman Holmberg, The God Ptah, Lund, 1946, p. 206-208; J. Berlandini, « Ptah-démiurge et l'exaltation du ciel », RdE 46 [1995], p. 20, n. 85; $L G G$ IV, 722-723) est bien attestée dans la région thébaine et à Karnak (par ex. Urk. VIII, n 208a; le roi est « Héritier de Tenen, semence divine de Celui qui est au Sud de son mur », Urk. VIII, n 197,10 ). Voir déjà pour la Deuxième Période Intermédiaire la stèle de donation au nom du souverain Séânkhi-Ptah, probablement d'origine thébaine : D. MEEKs, « Une stèle de donation de la Deuxième période intermédiaire ", ENiM 2 (2009), p. 129-137. Sur la stèle de Thoutmosis III (CGC 34013), l'épithète memphite est associée à Thèbes (infra) : «Ptah qui est au Sud de son mur dans Thèbes » (Urk. IV, 765, 5 et $7 ; 769,4$ et $8 ; 769,17 ; 770,2 ; L G G$ IV, 723c).

(b) Pour Ptah nhy nb rnp.wt, A. R. SChulman, « The God Nhj », JNES 23 (1964), p. 275-279; M. Sandman Holmberg, The God Ptah, p. 178-182 et p. 22 et 64 (nb rnp.wt); J.-Cl. GoYon, Confirmation du pouvoir royal au Nouvel An [Brooklyn Museum papyrus 47.218.50] (BdE 52), Le Caire, 1972, p. 66 (IX, 22), p. 99, n. 155 et Wilbour Monograph 7, New York, 1974, pl. VIII (col. 9, 22) :

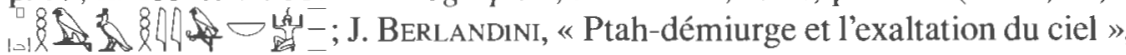
p. 13 et n. 29 (avec bibliographie); N. BEAUX, « La pintade, le soleil et l'éternité », p. $35 ; L G G$ III, 684 b.

(c) Ces deux dernières épithètes sont fréquentes pour Ptah memphite; voir respectivement $L G G$ II, $22 \mathrm{~b}$ et V, 375b-c. Pour « Ptah dont la puissance est grande ", M. Sandman Holmberg, The God Ptah, p. 111-112; P. Vernus, «Inscriptions de la Troisième Période Intermédiaire (III) », BIFAO 76 (1976), p. 10, n. g; J. Berlandin1, « Ptah-démiurge et l'exaltation du ciel », p. 15, n. 42-43; I. Guermeur, Les cultes d'Amon hors de Thèbes. Recherches de géographie religieuse (BEHE SR 123), Turnhout, 2005, p. 42 et n. f. Le « Grand trône » est associé à de nombreuses divinités, parmi lesquelles Horus de Béhédet et Ptah

4. Cf. N. BEAuX, « La pintade, le soleil et l'éternité », BIFAO 104 (2004), p. 23, fig. 2.

5. Cf. p. ex. Ph. Collombert, Le tombeau de Mérérouka, p. 113, 242, § 213. 
font figure de bénéficiaires favoris, d'après les occurrences réunies par le $L G G$; L. GABOLDE, « Un linteau tentyrite de Thoutmosis III dédié à Amon », BIFAO 99 (1999), p. 198-199; St. Pasqual, Topographie de Memphis la : Corpus. Temples et principaux quartiers de la XVIIf dynastie (CENiM 4), Montpellier, 2011, p. 14, 33, 58 (« dans le Grand trône de son père Ptah », ép. Horemheb) et 67.

\section{Commentaire}

Les pérégrinations de deux « naos à toit pyramidal » d'Amasis, originaires de Kôm el-Ahmar de la Menûfîyah (Louvre D 29 et Leyde AM 107), furent retracées au cours d'une minutieuse enquête menée par Jean Yoyotte, récipiendaire de ce volume ${ }^{6}$. En hommage « à celui qui a pu pénétrer les causes secrètes des choses » de l'antique Égypte, la publication de ce document vient compléter la longue liste des naos monolithes chers aux souverains de la XXvi ${ }^{e}$ dynastie $^{7}$ et étayer le dossier des monuments consacrés par le souverain saïte ${ }^{8}$.

6. J. Yoyotte, «Le Grand Kom el-Ahmar de Menufiyah et deux naos du Pharaon Amasis », BSFE 151 (2001), p. 54-83.

7. Pour une liste des naos monolithes, Chr. Thiers, « Un naos de Ptolémée Philadelphe consacré à Sokar ", BIFAO 97 (1997), p. 253-268 (en part. p. 261 pour Amasis); ajouter N. SPENCER, « The great naos of Nekhthorheb from Bubastis », EA 26 (2005), p. 21-24 (Nectanébo II); ID., A Naos of Nekhthorheb from Bubastis (BMRP 156), Londres, 2006 (en part. p. 21-22 pour Amasis) et p. 53-54 (autre fragment au nom de Nectanébo II); D. RosENow, «The Naos of "Bastet, Lady of the Shrine" from Bubastis », JEA 94 (2008), p. 247-266; P. WiLSon, The Survey of Saïs (Sa el-Hagar) 19972002 (MEES 77), Londres, 2006, p. 212-213 et pl. 30 (Amasis); A.-S. von BOMHARD, The Naos of the Decades (OCMA 3), Oxford, 2008 (Nectanébo I); F. ConTARDI, Il naos di Sethi I da Eliopoli : un monumento per il culto del dio Sole (CGT 7002) (CMET 12), Milan, 2009; Chr. FAVARD-MeEKs - D. Meers, « Les corps osiriens : du Papyrus du Delta au temple de Behbeit », dans L. Coulon (éd.), Le culte d'Osiris au Ir millénaire av. J.-C. (BdE 153), Le Caire, 2010, p. 48 et n. 59 (JE 43281 au nom d'Apriès et non Amasis; O. PERDU, « Neshor à Mendès sous Apriès », BSFE 118 [1990], p. 43, fig. 2b); P. SPENCER, « Digging Diary 2010 », EA 38 (2011), p. 26 (Alexandrie); voir également J. A. HARREL - V. M. Brown, "A Late-Period Quarry for Naoi in the Eastern Desert", EA 14 (1999), p. 18-20; H. VIRENQUE, « Les quatre Naos de Saft el-Henneh : un rempart théologique construit par Nectanebo I dans le Delta oriental », ÉA\&O 42 (2006), p. 19-28; D. KLotZ, « A Naos of Nectanebo I from the White Monastery Church (Sohag) », GM 229 (2011), p. 37-52.

8. Pour les monuments au nom d'Amasis, voir note précédente et infra, n. 27 (Karnak); GDG IV, p. 113-127; H. De Meulenaere, Herodotos over de $26^{\text {ste }}$ Dynastie $(I I, 147-I I I, 15)$ (BiMus 27), Louvain, 1951, p. 117-121 ; ID., LÄ I (1973), col. 182, s.v. " Amasis »; M. V. SETToNWilliams, « The Tell el-Farâ'în Expedition, 1968 », JEA 55 (1969), p. 7; A. I. Blöbaum, "Den ich bin ein König, der die Maat liebt”. Herrscherlegitimation im spätzeitlichen Ägypten. Eine vergleichende Untersuchung der Phraseologie in den offiziellen Königsinschriften vom Beginn der 25. Dynastie bis zum Ende der makedonischen Herrschaft (EgMon 4), Aix-la-Chapelle,

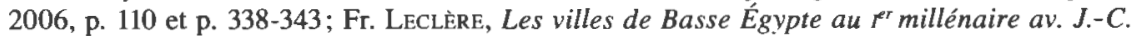
(BdE 144), Le Caire, 2008, en part. p. $62-64$ (Memphis), p. 172-177 (Saïs), 203-208 (Bouto), 245-246 (Athribis), 324-327 (Mendès), 493-494 (Bouto d'Arabie) et index p. 783; A. FARID, «Re-used Blocks from a Temple of Amasis at Philae: The Final Results », MDAIK 36 (1980), p. 81-103 (Philae); D. KLotz, « Two Studies on the Late Period Temples at Abydos ", BIFAO 110 (2010), p. 128-135; les stèles de donation sont réunies par D. MeEKs, "Une stèle de donation de la Deuxième période intermédiaire », p. 140 et 153-154; Pour le culte et le "souvenir » d'Amasis, voir P. WiLson, « A Cult of Amasis and "the procession of two Gods" at Saïs ", dans Th. Schneider - K. M. Szpakowska (éd.), Egyptian stories. Tribute to Alan B. Lloyd (AOAT 347), Münster, 2007, p. 444-449; Chr. ThiERs, « L'an 44 d'Amasis sur la grande stèle ptolémaïque 


\section{Christophe Thiers}

La face extérieure gauche polie assure que le naos n'était pas décoré sur les côtés (et à l'arrière), contrairement à certains monolithes, véritables temples miniatures, couverts de scènes sur les parois et les montants. La seule colonne de texte sur les montants va également dans ce sens ${ }^{9}$ et invite à considérer ce naos comme un monument « secondaire» par rapport à d'autres naos d'Amasis. On notera également qu'aucune trace ne témoigne de la présence d'un tore d'angle encadrant le monument.

L'absence de martelage du cartouche royal se doit d'être soulignée tant la damnatio memoriae qu'a subie le souverain saite est fréquente sur les monuments qui nous sont parvenus ${ }^{10}$. Naturellement, aucun indice de provenance du fragment n'apparaît dans le registre de la police. Les épithètes de Ptah conservées sur le montant appartiennent sans conteste au cercle memphite du dieu ( $r s y$-jnb $=f$, Tnn, Nhy). Parmi les monuments au nom d'Amasis, l'ensemble le plus notable est originaire de la région de Saqqara-Memphis, en lien étroit avec le temple de Ptah $^{11}$. On supposera pour autant que la plupart des objets saisis chez les antiquaires ou les receleurs de Louqsor proviennent de la région thébaine, en particulier pour les pièces les plus conséquentes, mais sans toutefois exclure une origine plus lointaine, plus précisément de la Haute Égypte.

Dans la région thébaine, Ptah fut vénéré en plusieurs endroits, notamment dans les temples funéraires de la rive ouest ${ }^{12}$; il est ainsi « celui qui réside à l'Ouest de Thèbes $»^{13}$. Sans entrer dans le détail de ses épithètes, notons que plusieurs de ses désignations spécifiquement memphites sont adaptées à Thèbes : «Ptah qui est au Sud de son mur dans Thèbes » (supra, n. a); « Maître de Ânkh-taouy dans Thèbes », « au beau visage dans Thèbes ». À l'image d'Amon-Rê, il devient « le chef de Thèbes » (hry-tp Wis.t $)^{14}$, et surtout « celui qui réside à Thèbes » (hry-jb

d'Héracléion ", dans D. Devauchelle (éd.), La xxvf dynastie : continuités et ruptures. Promenade saïte avec Jean Yovotte, Paris, 2011, p. 153-157.

9. Cf. le naos de Ptolémée Philadelphe Caire RT 16/8/34/1, Chr. THIERs, «Un naos de Ptolémée Philadelphe consacré à Sokar », p. 266-267.

10. D. KLotz, «Two Studies on the Late Period Temples at Abydos», p. 131, n. 33 (avec bibliographie).

11. A. I. Blöbaum, "Den ich bin ein König, der die Maat liebt", p. 340-341 ; Fr. Leclère, Les villes de Basse Égypte, p. 62-64.

12. Pour l'ensemble de la région thébaine, M. Sandman Holmberg, The God Ptah, p. 224-231. En l'an 1 de Horemheb (regravé sous Äy), la fête de Ptah à Karnak se tenait le 22 Khoiak (A. Mariette, Karnak. Étude topographique et archéologique, Leipzig, 1875, pl. 47d). Le calendrier des fêtes de Médinet Habou (EPIGraphic Survey, Medinet Habu III [OIP 23], Chicago, 1924, pl. 158 et 160) indique que Ptah-Sokar-Osiris du temple de Ramsès III était célébré du 21 au 30 Khoiak; M. Sandman Holmberg, The God Ptah, p. 229-230. Un graffite de Saqqara (KRIIII, 436, 12) mentionne la fête de Ptah qui est au Sud de son mur le 24 Khoiak. Dans ce contexte, pour Ptah et les rituels de Djêmé, Cl. Traunecker, « Les graffiti des frères Horsaïsis et Horemheb. Une famille de prêtres sous les derniers Ptolémées », dans W. Clarysse - A. Schoors - H. Willems (éd.), Egyptian Religion. The Last Thousand Years. Studies Dedicated to the Memory of Jan Quaegebeur 2 (OLA 85), Louvain, 1998, p. 1225; Chr. THIERS - Y. VoloKhine, Ermant I. Les cryptes du temple prolémaïque (MIFAO 124), Le Caire, p. 73-77. 13. $L G G \mathrm{~V}, 319 \mathrm{c}$ (« Ptah de la grande porte »).

14. $L G G \mathrm{~V}, 390 \mathrm{c}$. 
$W$ W. $t)^{15}$, également « celui qui préside à Thèbes » $(\text { hnty W3s.t })^{16}$ et « le maître de Thèbes » $(n b \text { Wss. } t)^{17}$. Il prend place dans le temple d'Amon-Rê en tant que « celui qui réside dans Karnak » $(h r y-j b J p . t-s . w t)^{18}$.

On signalera également l'existence de deux statues du Nouvel Empire (Amenhotep III) représentant le dieu Ptah et dont la provenance thébaine est admise. Découvertes par J. J. Rifaud, elles sont conservées au musée de Turin (C 86 et C 87) et figurent le dieu respectivement debout (granite) et assis (calcaire) $^{19}$; il porte les épithètes de "maître de Maât, grand dieu, maître de la fête-sed, qui réside dans le pavillon des annales " $\left(n b m 3^{\prime} . t n t r\right.$ 's $n b h b-s d h r y-j b$ $s h n g n . w t)^{20}$ et de celui « qui préside au Château de l'or » $(h n t y / n h w . t-n w b)^{21}$. Le groupe statuaire d'un pharaon agenouillé devant le dieu, découvert dans le temple de Ptah à Karnak, ne porte malheureusement aucune inscription ${ }^{22}$.

C'est en effet dans ce dernier temple, bâti à l'extérieur du domaine d'Amon-Rê puis englobé par l'enceinte de la $\mathrm{XXX}^{\mathrm{e}}$ dynastie $^{23}$, que le culte de Ptah s'est principalement développé. Pourtant, rien ne vient étayer, dès l'abord, la possibilité que le naos provienne de cet édifice situé au nord du domaine amonien. Les épithètes locales les plus en usage du dieu memphite sont celles de "maître de Thèbes / qui réside à Thèbes » et celle commune de «maître de Maât » ${ }^{24}$, absentes du fragment du naos. Rien n'empêcherait de supposer, toutefois, que ces caractéristiques thébaines aient été inscrites sur le montant droit. Rappelons que la stèle de la Deuxième Période Intermédiaire, dont il a été question précédemment et qui

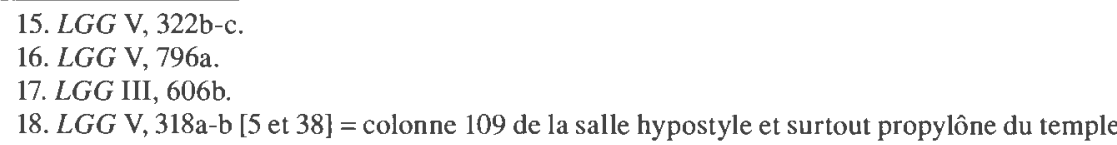
de Ptah (Ptolémée Philométor) : nb Wis.t hry-jb Jp.t-s.wt (Urk. VIII, n ${ }^{\circ} 188 \mathrm{~b}$ ); pour le lien entre la porte nord de la salle hypostyle et le temple de Ptah vers lequel elle menait, EPIGRAPHIC SuRvey, Reliefs and Inscriptions at Karnak IV (OIP 107), Chicago, 1986, pl. 19 (right et left : Ptah et Sekhmet sur les premiers registres); Chr. Loeben, « À propos de la graphie du nom de Séthi I à Karnak », CahKarn VIII (1985), p. 227.

19. $\mathrm{PM} \mathrm{II}^{2}$, p. 215 ; A. M. DONADONI-ROVERI (dir.), La civilisation des Égyptiens. Les arts de la célébration, Turin, 1989, p. 105 (C 86) et 141 (C 87); E. SCamuzzi, Lart égyptien au Musée de Turin, Turin, 1966, pl. 23 et p. 2, n. 1 (C 86). Voir ici même, J.-J. Fiechter, « Rifaud à Thèbes. 1816-1822 », p. 451. On sait que J.-J. Rifaud découvrit dix-neuf statues en granite et une en calcaire dans le temple de Ptah dont il fut le premier à explorer les ruines; Rapport de la Commission nommée pour l'examen de la collection des dessins de $M$. Rifaud sur l'Égypte et la Nubie, Paris, 1829, p. 19; d'après P. BARguet, Le Temple d'Amon-Rê à Karnak. Essai d'exégèse (RAPH 20), Le Caire, 1962, p. 13, n. 6. On signalera également huit statues et une stèle consacrées à Ptah découvertes par Georges Legrain dans la cour de la Cachette (http:// www.ifao.egnet.net/bases/cachette/, s.v. Ptah) ainsi que la statue CGC 38068 (PM II ${ }^{2}$, p. 285) et le « naos » d'Aménémopé CGC 70026 (G. RoEDER, Naos [CGC 70001-70050], Leipzig, 1914, pl. 36 et p. 105-106).

20. Urk. IV, 1756 (596)

21. Urk. IV, 1755 (595).

22. Photographie récente dans Chr. Thiers - P. Zignani, « The Temple of Ptah at Karnak », EA 38 (2011), p. 24.

23. J.-Cl. Golvin - El-S. Hegazy, «Essai d'explication de la forme et des caractéristiques générales des grandes enceintes de Karnak ", CahKarn IX (1993), p. 146; Chr. ThIERs P. Zignani, « The Temple of Ptah at Karnak », p. 21.

24. $L G G$ III, $639 \mathrm{c}-640$. 
pourrait être originaire de la région thébaine, est adressée à « Ptah qui est au Sud de son mur $»^{25}$.

En outre, on soulignera que les souverains de la XXvi $\mathrm{f}^{\mathrm{e}}$ dynastie sont absents du temple de Ptah. Après l'adjonction de deux portes d'enceinte sous le règne de Chabaka, l'arasement de ses cartouches par Psammétique II selon toute probabilité et le travail de Takélot sur la porte principale d'accès au temple, aucune activité architecturale ou décoratrice n'est attestée jusqu'aux remaniements opérés sous Ptolémée Évergète Ir et Ptolémée Philopator ${ }^{26}$. On ajoutera qu'Amasis n'est que rarement attesté dans la grande enceinte d'Amon-Rê $\hat{e}^{27}$.

Sans autoriser une reconstitution sûre du naos, la hauteur du fragment conservé $(1,73 \mathrm{~m})$ permet d'avoir une idée relativement précise des dimensions du monument. En supposant que seul le signe mry («aimé de ») manquait en fin de colonne, on peut évaluer la hauteur du montant à environ $1,80 \mathrm{~m}$. Si l'on conserve des proportions par comparaison avec d'autres naos monolithes, il n'est pas impossible que le naos d'Amasis mesurât entre 2,50 et 2,80 m de haut pour 1,30 à $1,70 \mathrm{~m}$ de large ${ }^{28}$. De telles dimensions excluent de placer ce monument à l'intérieur du temple de Ptah à Karnak, la porte d'accès à la chapelle centrale, dont les reliefs ont été restaurés à l'époque ptolémaïque, étant large de 1,21 m, le passage de celle de la chapelle nord consacrée à Ptah mesurant quant à lui $1,04 \mathrm{~m}^{29}$. On soulignera qu'aucun indice architectural ne vient attester le possible démontage de la paroi arrière du temple, pratique dont témoigne, par exemple, le petit temple de Médinet Habou ${ }^{30}$.

25. D. Meeks, « Une stèle de donation de la Deuxième période intermédiaire », p. 129-137.

26. Chr. Thiers - P. Zignani, « The Temple of Ptah at Karnak », p. 20-24.

27. Chapelle d'Osiris Ounnefer Neb-djefaou consacrée par Ânkhnesneferibrê et Amasis :

L. Coulon - C. Defernez, «La chapelle d'Osiris Ounnefer Neb-Djefaou à Karnak », BIFAO 104 (2004), p. 135-190; chapelle d'Osiris maître de l'éternité consacrée par Ânkhnesneferibrê, Amasis et Psammétique III : Cl. Traunecker, « La chapelle d'Osiris "seigneur de l'éterniténeheh" à Karnak ", dans L. Coulon (éd.), Le culte d'Osiris au fer millénaire av. J.-C., p. 155-194; deux montants de porte d'Amasis (palimpsestes) au sud-est du lac sacré (consacrés à AmonRê) : A. Masson - M. Mtllet, dans M. Boraik - Chr. Thiers, Centre franco-égyptien d'étude des temples de Karnak. Rapport 2008, Louqsor, 2009, p. 17 (http://www.cfeetk.cnrs.fr/fichiers /Documents/Rapport-2008-FR.pdf). Voir également le scellé du vizir Psammétique-sa-Neith, associé au cartouche d'Amasis découvert dans le quartier des prêtres : A. MAsson, « Un scellé du vizir Psametik-sa-Neith », CahKarn XII (2008), p. 657-658; L. Coulon - A. MAsson, "Osiris Naref à Karnak », dans L. Coulon (éd.), Le culte d'Osiris au $\ell^{r}$ millénaire av. J.-C., p. 140-141. On signalera à titre indicatif une stèle de donation conservée à Karnak mais adressée à Isis de Chemnis, dont l'étude est en cours par les soins de Dimitri Meeks (CFEETK, nos 114836 et 1430); voir D. MeEKS, « Une stèle de donation de la Deuxième période intermédiaire », p. 153 (26.5.1c).

28. La hauteur $(2,50 \mathrm{~m})$ serait identique à celle du naos du Louvre D 29; J. YoYotTe, "Le Grand Kom el-Ahmar de Menufiyah et deux naos du Pharaon Amasis ", p. 68 et 67, fig. 6.

29. Relevés architecturaux réalisés sous la direction de P. Zignani (CFEETK-USR 3172 du CNRS).

30. G. DaRessy, «Comment fut introduit le naos du petit temple de Médinet Habou », RT 22 (1900), p. 144-146; en dernier lieu, EpIGRaphic Survey, Medinet Habu IX. The Eighteenth Dynasty Temple I (OIP 136), Chicago, 2009, p. 38 et n. 20 (démontage qui pourrait dater de Ptolémée Sôter II ou antérieurement). Le démantèlement de la paroi orientale de la chapelle d'Alexandre dans le temple de Karnak ne semble pas ressortir de cette même nécessité pratique dans la mesure où la statue de faucon en calcaire installée dans cet espace a pu y accéder 
Si Amasis orna plusieurs temples du Delta de naos monolithes, en Haute Égypte seul le temple d'Abydos semble témoigner d'une telle attention ${ }^{31}$. À considérer au mieux qu'il soit thébain, le montant de naos saisi par la police de Lougsor apporterait un témoignage supplémentaire de l'intérêt tout relatif d'Amasis à l'égard des sanctuaires du Saïd ${ }^{32}$. Pour autant, les seuls indices memphites du montant conservé ne permettent pas de l'attribuer à un temple précis, le temple de Ptah à Karnak, trop exigu, ne pouvant l'abriter.

par la porte d'entrée occidentale; pour les modifications architecturales de ce sanctuaire, J.-Fr. CarlotTr, LAkh-menou de Thoutnosis III à Karnak. Érude architecturale, Paris, 2001, p. 122-126; Ph. MARTINEZ, «À propos de la décoration du sanctuaire d'Alexandre à Karnak », BSEG 13 (1989), p. 107-116.

31. Chr. Thiers, « Un naos de Ptolémée Philadelphe consacré à Sokar », p. 261 (9); pour un naos remarquable érigé à Saïs, Hérodote II, 175; «Dans tous les autres temples de quelque renom, Amasis fit élever également des ouvrages remarquables par leur grandeur », Hérodote II, 175 (traduction d'A. Barguet, Héradote, La Pléiade, Paris, 1985). Le temple de Philae dont les blocs attestent l'existence (supra, n. 8) devait également être doté d'un naos.

32. Le soutien thébain à Apriès serait à l'origine de ce désintérêt mais surtout de la réorganisation administrative (introduction du démotique) et de l'affaiblissement du pouvoir des Grands majordomes des divines adoratrices; A. LEAHY, «The Earliest Dated Monument of Amasis and the End of the Reign of Apries ", JEA 74 (1988), p. 197-198. 

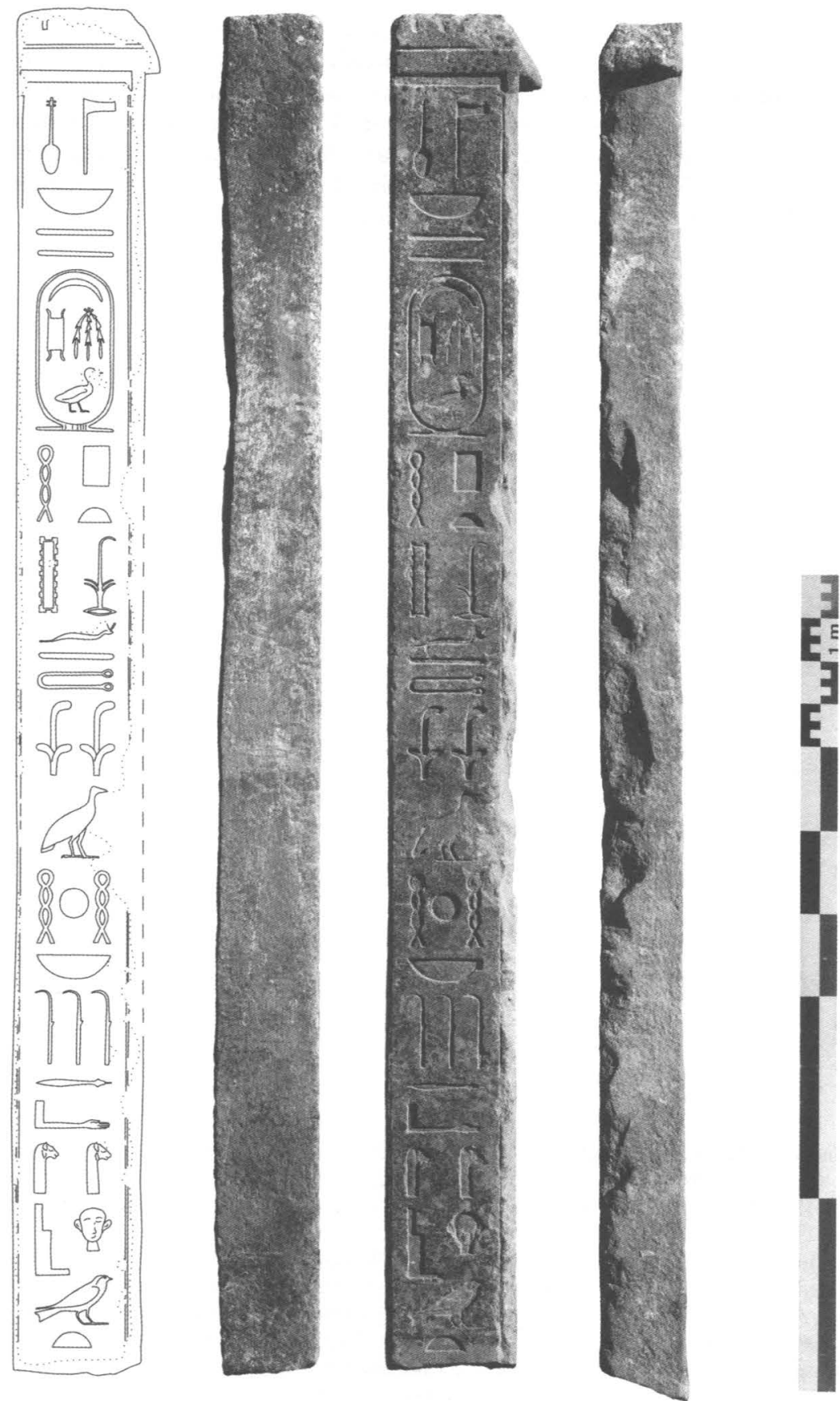

P1. 1. Fac-similé et photographies du montant du naos d'Amasis (C) Cnrs-Cfeetk nos $135337-135339 / \mathrm{S}$. Klein, K. Dowi). 


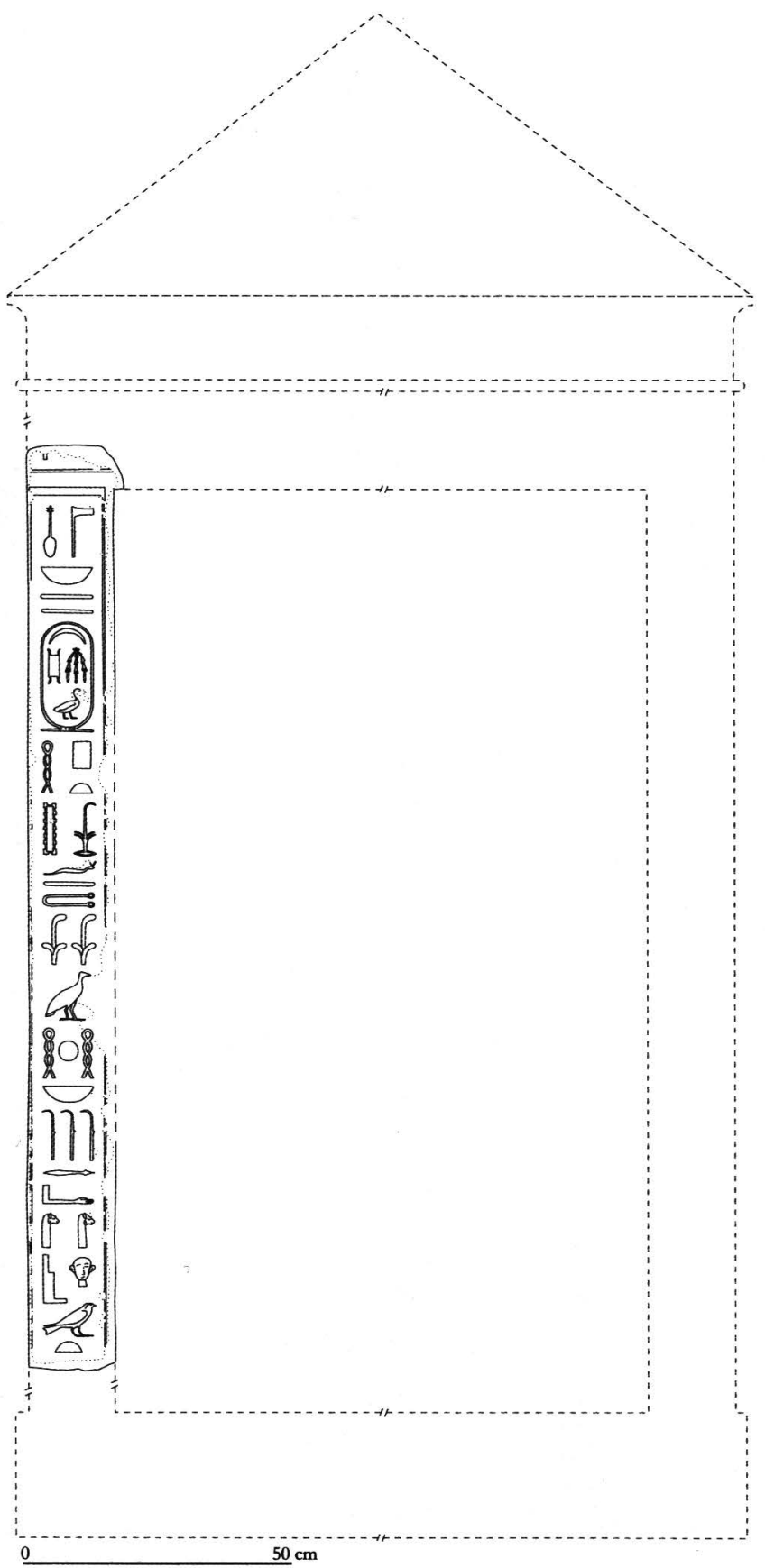

P1. 2. Proposition de restitution du naos d'Amasis (C) Cnrs-Cfeetk/Chr. Thiers). 